\title{
An in vitro Comparison between Two Methods of Electrical Resistance Measurement for Occlusal Caries Detection
}

\author{
J. Kühnisch ${ }^{a}$ R. Heinrich-Weltzien ${ }^{b}$ M. Tabatabaie ${ }^{b}$ L. Stösser ${ }^{b}$ \\ M.C.D.N.J.M. Huysmans ${ }^{c}$ \\ ${ }^{a}$ Department of Conservative Dentistry and Periodontology, Ludwig Maximilians University, Munich, \\ ${ }^{b}$ Department of Preventive Dentistry, Friedrich Schiller University, Jena, Germany; ${ }^{\mathrm{c}}$ Department of Dentistry, \\ University Medical Centre Groningen, Groningen, The Netherlands
}

\author{
Key Words \\ Occlusal caries diagnosis - Electronic caries monitor • \\ ECM III - Cariometer · CRM $800 \cdot$ Reproducibility · \\ Diagnostic performance
}

\begin{abstract}
Because of different measurement techniques and the easier design of the CRM prototype, this in vitro study aimed to compare the diagnostic performance and reproducibility of two electrical methods (Electronic Caries Monitor III, ECM and Cariometer 800, CRM) for occlusal caries detection, and to evaluate the effect of staining/ discoloration of fissures on diagnostic performance. Hundred and seventeen third molars with no apparent occlusal cavitation were selected. Six examiners inspected all specimens independently, using the CRM, and a subgroup of 4 using the ECM. Histological validation using a stereomicroscope was performed after hemisectioning. Intra- and interexaminer reproducibility was assessed by Lin's concordance correlation coefficient (CCC) and Bland and Altman analysis. Diagnostic performance parameters included sensitivity (SE), specificity (SP) and area under the ROC curve $\left(A_{z}\right)$. The CCC yielded an intraand interexaminer reproducibility of $0.69 / 0.62$ (ECM) and of $0.79 / 0.74$ (CRM). The mean intra- and interexaminer
\end{abstract}

95\% range of measurements (range between Bland and Altman limits of agreement) given in percentages of the instrument reading were $67 \% / 65 \%$ for the ECM and $28 \% / 33 \%$ for the CRM. $A_{z}$ at the D3-4 level was 0.74 (ECM) and 0.78 (CRM). The CRM showed at least equivalent diagnostic performance to the ECM. However, improvement is still desirable. Diagnostic performance appeared to be enhanced in discolored lesions; however, this may be related to sample lesion distribution characteristics.

Copyright $(2006$ S. Karger AG, Basel

Occlusal caries has been changing not only in prevalence but also in pattern and progression, and the correct classification of occlusal caries lesions has become more difficult. This is one of the reasons for the call for better diagnostic tools over the past 10 years [Wenzel et al., 1991; Verdonschot et al., 1993a; Pine and ten Bosch, 1996; Pitts, 2001]. There is agreement that the optimal technique should have good diagnostic performance, be noninvasive, allow a valid quantification of disease progression and an objective lesion monitoring, and easy to carry out at reasonable costs. The accurate detection of sound and cavitated surfaces usually does not constitute a major problem. However, noncavitated lesions remain

\section{KARGER}

Fax +4161306 1234 E-Mail karger@karger.ch www.karger.com 
a challenge. The evaluation of newly developed diagnostic methods should focus on this particularly difficult situation [Pitts and Stamm, 2002].

Electrical resistance measurement is one of the techniques proposed for occlusal caries lesion detection. It depends on the permeability changes due to caries demineralization of the tissues. The present standard instrument is the Electronic Caries Monitor (ECM), which is used almost exclusively by research groups. The basic measurement technique is a measurement of the resistance at a site in the fissure, using a probe with a coaxial airflow in order to dry the tissue around the probe and to prevent current leakage. Several alternative measurement protocols have been suggested and explored with this instrument, including application of conductive gel [Huysmans et al., 1998a; Ricketts et al., 1997c], varying the airflow and resistance calculation [Ricketts et al., 1997a, b], and using complex outcome parameters [Ellwood and Côrtes, 2004]. Two alternative instrument types have been described. The Cariometer 800 (CRM prototype) is a much simpler instrument, using brief air drying before the measurement instead of coaxial airflow drying. This enables the instrument to scan fissures, instead of making multiple site measurements. It measures resistance at a higher frequency than the ECM: $500 \mathrm{~Hz}$ instead of $23 \mathrm{~Hz}$ [Schulte and Pieper, 1997]. A more complicated measurement technique has been described, based on the principle of electrical impedance spectroscopy (EIS) [Longbottom et al., 1996].

Both details of the measurement technique and the actual measurement protocol may have a profound influence on the diagnostic performance of electrical measurements. EIS is assumed to be a more appropriate technique than single frequency measurements. However, up to now, the superior diagnostic performance of EIS-based instruments has not been demonstrated. The single frequency at which measurements are made may also influence the resistance estimate of the instrument. Whereas a low frequency may give rise to polarization effects (overestimating resistance), a higher frequency may underestimate true direct-current resistance [Huysmans, 2000].

The measurement technique has been varied throughout the research into electrical detection of caries lesions. The main focus should be the method of achieving good contact without current leakage to the gingivae. The airflow method has the disadvantage of taking several seconds for each measurement site. Application of a conducting medium to the whole occlusal surface after drying would also take time, and has the added disadvantage that the exact site of the lesion in the fissure system is not detected. Drying the tooth surface initially and then scanning it by moving the instrument probe along the fissure system would be an easier procedure if good probe contact could be achieved.

From previous studies it can be concluded that the ECM may be a valuable adjunct to visual inspection for suspect occlusal fissures [Verdonschot et al., 1993b; Lussi et al., 1995; Ricketts et al., 1996; Ekstrand et al., 1997; Huysmans et al., 1998b; Pereira et al., 2001; Bamzahim et al., 2002]. However, the method has not reached general dental practice, which may be due to the time-consuming measurement protocol and the high cost of the equipment. If a simpler measurement protocol, using a much cheaper instrument, yielded the same or better diagnostic performance, the method would perhaps be more generally applicable.

It was the aim of this study to compare the diagnostic performance and reproducibility of the ECM III and the CRM prototype in vitro, in a clinically relevant sample of molar teeth without gross cavitations. Because a recent report indicated that the performance of the ECM is compromised in teeth with stains and/or discolored lesions [Côrtes et al., 2003], we also evaluated the performance for unstained and discolored subgroups.

\section{Materials and Methods}

A sample of 117 sound and noncavitated third molars was selected from a pool of teeth extracted for surgical or orthodontic reasons. Only molars with complete root formation and without any sealants, fillings, gross occlusal cavitations, approximal and/or buccal/lingual caries lesions were included.

After gross debris had been removed, the teeth were cleaned with rotating brushes and an air polishing device (Air Flow, EMS, Switzerland). The roots of each tooth were completely embedded in cold-polymerizing methacrylate blocks (Speiko, Münster, Germany) and the teeth were coded. A hole with a diameter of $\sim 5 \mathrm{~mm}$ was drilled in the resin block to the root, to ensure electrical contact during the measurements. To prevent bacterial growth, all specimens were stored in individual containers with physiological saline containing $0.02 \%$ sodium azide. Prior to the study, color photographs of the occlusal surfaces were made to assist in the histological validation later on.

Six examiners carried out the study, all 6 performing CRM measurements, and 4 performing ECM measurements. All examiners were trained in using both instruments before the study. The complete sample was measured twice by each examiner, to determine intra-examiner reproducibility. The measurement series were carried out with at least a week interval to ensure examiners would not recall their earlier findings. For each measuring cycle all teeth were collected in a large saline-filled bowl and only taken out for the electrical resistance measurement to limit dehydration. All measurement results were recorded by an assistant. 


\section{Measurements with the ECM}

The ECM III model (Lode Diagnostics, Groningen, The Netherlands) was used. Each tooth was positioned in a petri dish with physiological saline in such way that the top of the embedding material covering the root emerged from the liquid. Bubbles in hole were completely removed before measuring. The reference electrode was put into contact with the saline in the petri dish. When the instrument probe touched the occlusal fissure, the coaxial airflow $(7.5$ liters $/ \mathrm{min}$ ) was automatically activated. After $5-10 \mathrm{~s}$, the stable resistance was read from the instrument panel, showing a value between 0 and $100 \mathrm{M} \Omega$ (table 1). The measurement was performed at all suspect sites (e.g. deep pits and fissures, discolorations, opacities, microcavities, etc.) and repeated when a measurement was in doubt. The lowest resistance for each tooth was recorded. All examiners were instructed to moisten the tooth surface every $30 \mathrm{~s}$ or before every third measurement (rewetting).

\section{Measurements with CRM}

The CRM instrument (Cariometer 800; University of Marburg, Germany) consisted of a handpiece with the instrument probe, connected to a standard multimeter (VC404, Voltcraft, Hirschau, Germany). The sharp probe tip consisted of a thin metal tube (internal diameter $\sim 0.5 \mathrm{~mm}$ ). The handpiece included an array of LED lights which were selectively activated, depending on the measured resistance, ranging from 1: green (high resistance, sound) to 10: red (low resistance, deep lesion). The resistance range covered by these scores was 0-2 $\mathrm{M} \Omega$ (table 1). After positioning the tooth and the reference electrode in the petri dish in the same way as described for the ECM, the occlusal surface was dried with an air syringe for $\sim 1 \mathrm{~s}$. The entire fissure system was then scanned with the instrument probe. The instrument probe was dipped once into saline before the measurement, to fill the capillary tube with conducting fluid, thus promoting electrical contact. For each tooth, the maximum LED reading was recorded.

\section{Histological Validation}

Each tooth was hemisectioned mesiodistally for histological validation, using a microtome saw of $200 \mathrm{~mm}$ thickness (Mikrotrenn MT 1557, Hofer, Switzerland). The location of the cut was determined by visual assessment of the color photographs, selecting the site with the highest probability of caries lesions (e.g. deepest point of the fissure system, presence of discolorations, opacities, microcavities). Additional sections were made in cases where not all caries lesions or not the greatest lesion extension was deemed to be included in the section.

The sections were viewed under a stereomicroscope (Stemi SV11, Zeiss, Oberkochen, Germany) at $16 \times$ magnification. Enamel caries was defined histologically as opacity. Brown/yellow discolorations of dentin were associated histologically with dentin caries. Validation ratings were obtained for all sites on a scale from 0 to 4 : D0 $=$ no lesion, D1/2 = lesion in the enamel, D3 = lesion up to half the dentin, and D4 = lesion beyond half the dentin. The deepest lesion found in the sections of one tooth was recorded as the histological lesion depth for that tooth. Histological validation was performed by one examiner, who consulted a second examiner in cases of doubt.

The photographs were also used to determine the color status of the occlusal surfaces. The photographs were assessed by two examiners, and a consensus score was given to each surface: 0 for no staining or discoloration; 1 for staining (narrow brown line at base of fissure) only; 2 for discolored lesions.
Table 1. Cut-offs for occlusal caries lesion detection of the two devices for electrical resistance measurement, according to the manufacturers' instructions [Lode Diagnostics, 1996; Gente 1999]

\begin{tabular}{lcll}
\hline \multirow{2}{*}{$\begin{array}{l}\text { Determined caries } \\
\text { extension }\end{array}$} & \multirow{2}{*}{$\mathrm{ECM}$ range } & \multicolumn{2}{l}{ CRM range } \\
\cline { 3 - 4 } & $\mathrm{M} \Omega$ & $\mathrm{LED}$ & $\mathrm{M} \Omega$ \\
\hline Sound fissure (D0) & $10.00-99.99$ & $1-3$ & $2.000-1.142$ \\
Enamel caries (D1-2) & $4.00-17.00^{1}$ & $4-6$ & $1.141-0.486$ \\
& $1.50-6.00^{2}$ & & \\
Dentinal lesion (D3) & $1.00-2.00$ & 7,8 & $0.485-0.207$ \\
Deep dentinal caries (D4) & $<1.50$ & 9,10 & $0.206-0.000$ \\
\hline
\end{tabular}

${ }^{1}$ Initial caries. ${ }^{2}$ Enamel caries.

\section{Data Analysis}

Data were analyzed using SPSS 10.0 (SPSS Inc., Chicago, Ill., USA) and Excel 2000 (Microsoft corporation, Redmond, Wash., USA). Lin's concordance correlation coefficient (CCC) was used to measure the intra- and inter-examiner reproducibility of the ECM and CRM measurements [Lin, 1989]. Analysis by the method of Bland and Altman [1986] was used to look for systematic differences and to calculate $95 \%$ limits of agreement for each method, which were compared with the available measurement range [Axmann et al., 2002].

Spearman rank correlation with histological lesion depth and ROC analysis were used to assess the overall diagnostic performance of ECM and CRM. Plots of sensitivity (SE) and specificity (SP) were also constructed. Maximum values of SE + SP were used to determine optimal cut-off points for lesion detection. If several cut-offs resulted in the same maximum $\mathrm{SE}+\mathrm{SP}$, the cut-off with the highest SP was chosen. These analyses were repeated for all included subgroups.

\section{Results}

Histological validation revealed that 13 teeth $(11 \%)$ showed no signs of demineralization, 47 teeth $(40 \%)$ had a caries in the enamel (D1-2), 36 teeth (31\%) showed a lesion limited to the outer half of the dentin (D3), whereas in 21 teeth $(18 \%)$ caries had already spread into the inner half of the dentin (D4). The overall caries prevalence rate in the sample was $88 \%$ (D1-4 threshold) and $49 \%$ dentin caries prevalence (D3-4 threshold). As the overall prevalence of caries was so high, the analyses of diagnostic performance were only performed for caries at the dentin level.

Preliminary evaluation of the CRM data showed that errors had occurred for the first measurement cycle for examiners 1,2 and 4: the multimeter was probably scaled incorrectly. These examiners were excluded from all sub- 

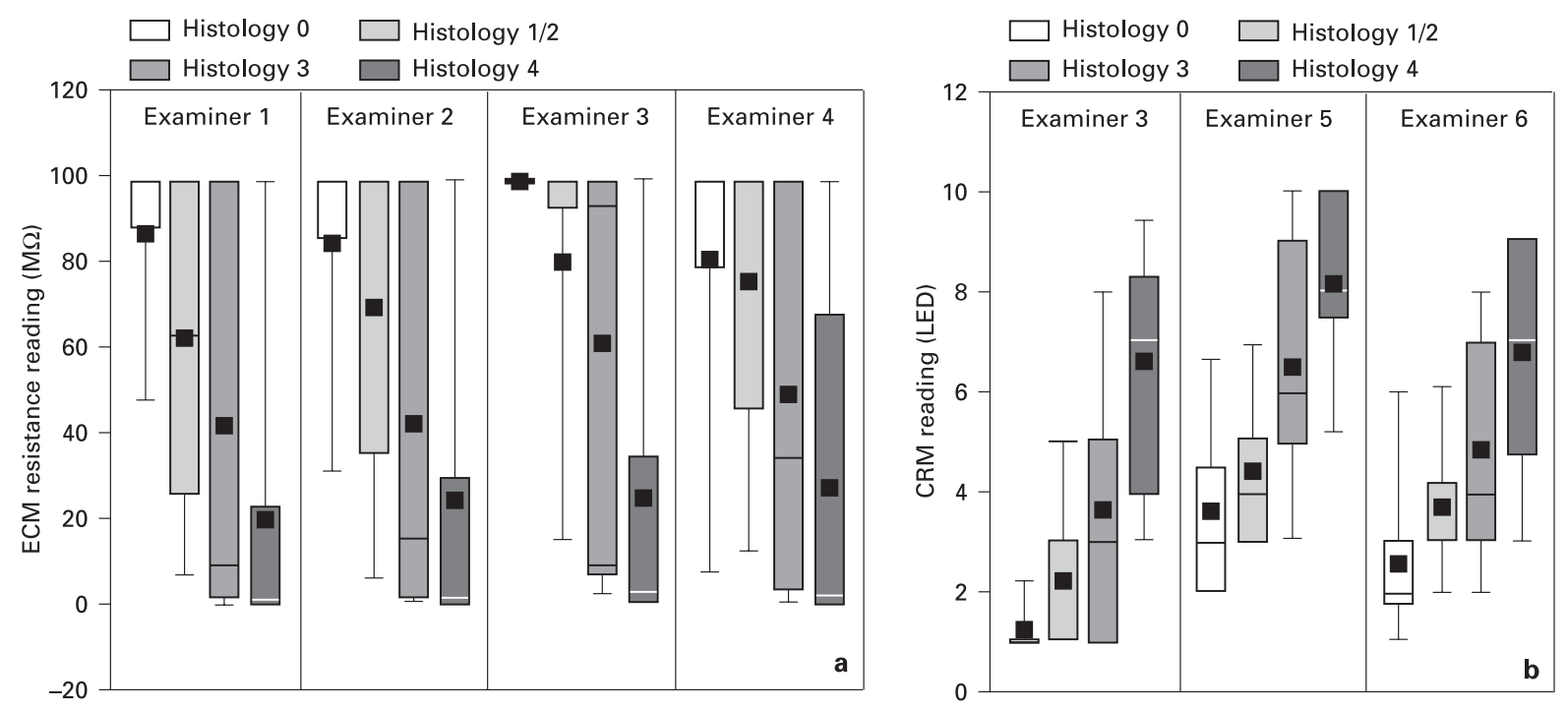

Fig. 1. Box plots of electrical resistance measurements vs. histological lesion depth for all examiners separately for ECM (a) and CRM (b). The line indicates the median, the box the 25th and 75th percentiles, and the whiskers the 10th and 90th percentiles. The mean is shown as a gray square for extra information.

sequent CRM analyses. Box plots for the results of ECM and CRM measurements in relation to histological lesion depth can be seen in figure 1 .

\section{Intra- and Inter-Examiner Reproducibility}

The results of the reproducibility analyses can be seen in table 2. The CCC revealed an average intra- and interexaminer reproducibility of $0.69 / 0.62$ for the ECM and of $0.79 / 0.74$ for the CRM. The mean intra- and inter-examiner $95 \%$ range of measurements (range between Bland and Altman limits of agreement given in units of the instrument reading) were 134/130 for the ECM and 5.5/6.6 for the CRM. This constituted $67 \% / 65 \%$ of the maximum variation for the ECM, and 28\%/33\% for the CRM.

\section{Diagnostic Performance}

ROC curves for all examiners and both methods are shown in figure 2. Correlation with histology and area under the ROC curve $\left(A_{z}\right)$ were in the same range for all examiners and both methods. The mean correlation with histology was -0.50 (range -0.46 to -0.53 ) for the ECM and 0.61 (range $0.60-0.62$ ) for the CRM. The correlation for the ECM is negative, as resistance is inversely related to lesion formation, whereas the CRM is not. Corre-
Table 2. Reproducibility of the electrical resistance measurements for all examiners

\begin{tabular}{|c|c|c|c|c|}
\hline & \multicolumn{2}{|c|}{$\begin{array}{l}\text { Lin's concordance } \\
\text { correlation coefficient }\end{array}$} & \multicolumn{2}{|c|}{$\begin{array}{l}\text { Bland and Altman's } \\
\text { limits of agreement }\end{array}$} \\
\hline & $\mathrm{CCC}_{\text {Intra }}$ & $\mathrm{CCC}_{\text {Inter }}$ & range $_{\text {intra }}$ & range $_{\text {inter }}$ \\
\hline ECM, mean & 0.69 & 0.62 & 134 & 130 \\
\hline Examiner 1 & 0.72 & 0.69 & 125 & 122 \\
\hline Examiner 2 & 0.65 & 0.60 & 145 & 132 \\
\hline Examiner 3 & 0.75 & 0.56 & 120 & 139 \\
\hline Examiner 4 & 0.64 & 0.62 & 145 & 127 \\
\hline CRM, mean & 0.79 & 0.74 & 5.5 & 6.6 \\
\hline Examiner 3 & 0.73 & 0.74 & 6.0 & 6.9 \\
\hline Examiner 5 & 0.80 & 0.73 & 5.0 & 6.2 \\
\hline Examiner 6 & 0.83 & 0.75 & 5.6 & 6.6 \\
\hline
\end{tabular}

sponding $\mathrm{A}_{\mathrm{z}}$ values were 0.74 (ECM, range 0.73-0.75) and 0.78 (CRM, range $0.75-0.80)$. Clear optimal cut-offs were not obvious in most examiners for either device

\section{Stained and/or Nondiscolored Fissures}

The division of the sample into staining subgroups resulted in histology distributions as shown in table 3. As 


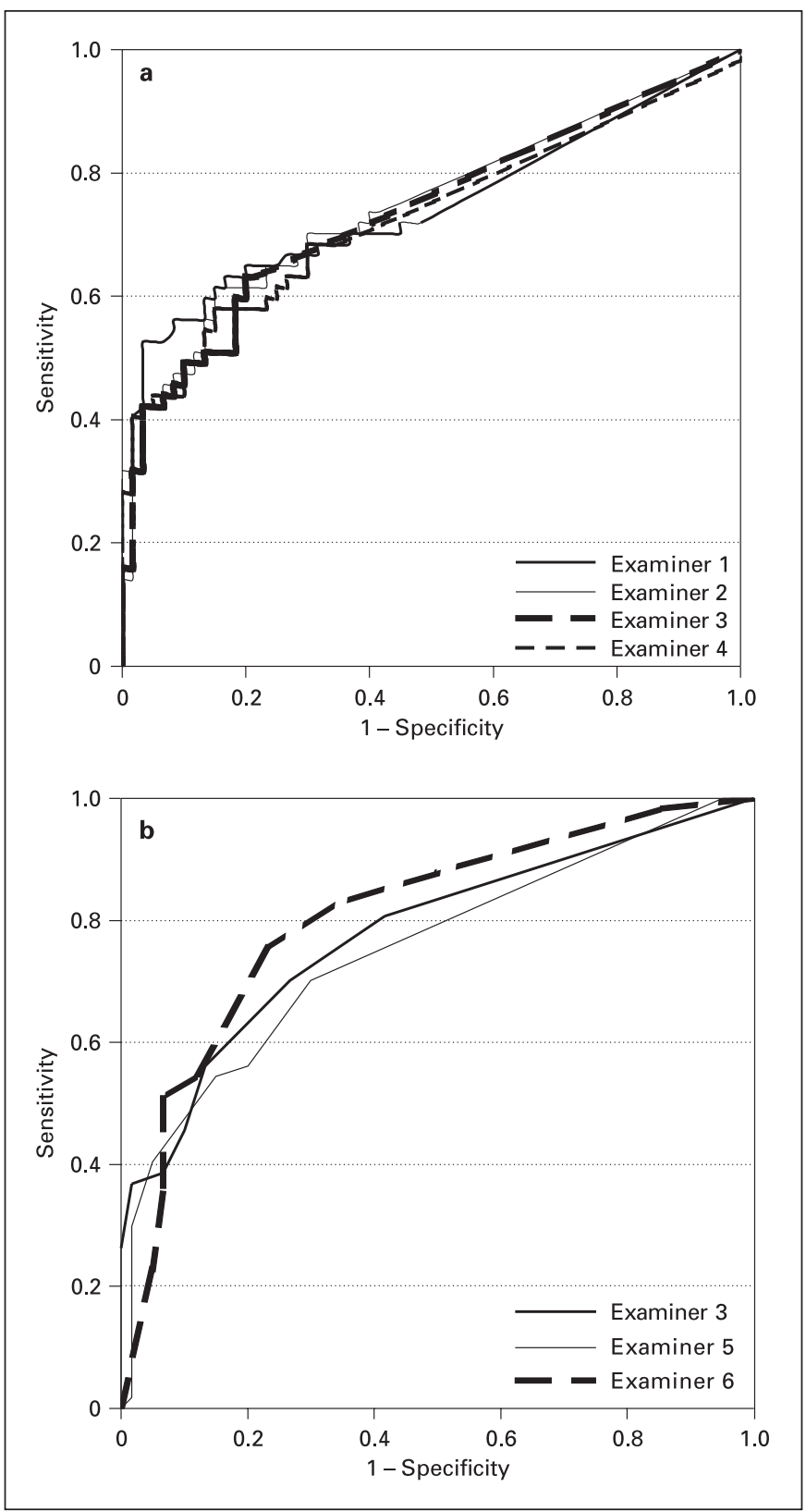

Fig. 2. ROC curves of all examiners for ECM (a) and CRM (b) at the D3-4 level.

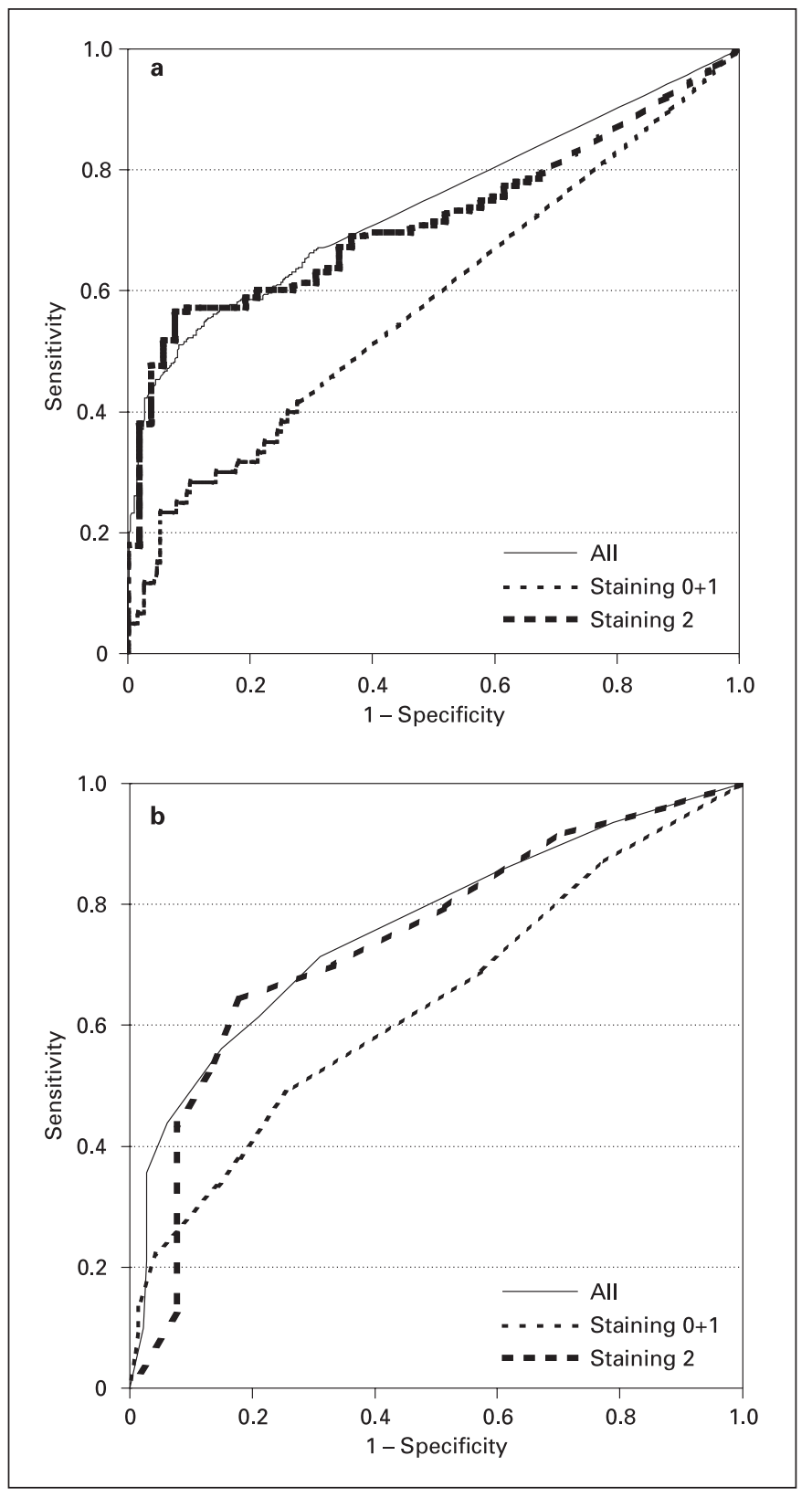

Fig. 3. Average ROC curves for ECM (a) and CRM (b) at the D34 level, for all teeth and for unstained and stained (groups 0 and 1) and brown discolored fissures (group 2) separately.
Table 3. Distribution of lesion severity, relative to staining and brown discolorations of the occlusal surface

\begin{tabular}{lrrrrr}
\hline \multicolumn{2}{l}{ Histology } & & & \\
\cline { 2 - 6 } & D0 & D1-2 & D3 & D4 & $\Sigma$ \\
\hline No discoloration/no staining (0) & 8 & 32 & 12 & 1 & 53 \\
Staining only (1) & 3 & 4 & 2 & 0 & 9 \\
Brown discoloration (2) & 2 & 11 & 22 & 20 & 55 \\
$\Sigma$ & 13 & 47 & 36 & 21 & 117 \\
\hline
\end{tabular}


Table 4. Diagnostic performance for the D3-4 level of the two devices for electrical resistance measurements on occlusal surfaces for the complete sample and staining/discoloration subgroups $(0=$ no stain/discoloration, $1=$ staining only, $2=$ brown discoloration $)$

\begin{tabular}{|c|c|c|c|c|c|c|c|c|c|c|c|c|}
\hline \multirow[t]{3}{*}{ Staining } & \multicolumn{6}{|l|}{ ECM } & \multicolumn{6}{|l|}{ CRM } \\
\hline & \multirow{2}{*}{$\begin{array}{l}\text { correlation } \\
\text { with } \\
\text { histology }\left(r_{\mathrm{s}}\right)\end{array}$} & \multicolumn{2}{|c|}{$\mathrm{ROC}$} & \multicolumn{3}{|c|}{ optimal performance } & \multirow{2}{*}{$\begin{array}{l}\text { correlation } \\
\text { with } \\
\text { histology }\left(r_{s}\right)\end{array}$} & \multicolumn{2}{|c|}{ ROC } & \multicolumn{3}{|c|}{ optimal performance } \\
\hline & & $\mathrm{A}_{\mathrm{z}}$ & $\begin{array}{l}\text { standard } \\
\text { error }\end{array}$ & SE & SP & $\mathrm{M} \Omega$ & & $\mathrm{A}_{\mathrm{z}}$ & $\begin{array}{l}\text { standard } \\
\text { error }\end{array}$ & SE & SP & LED \\
\hline All & -0.50 & 0.74 & 0.02 & 0.57 & 0.85 & $20-25$ & 0.61 & 0.78 & 0.04 & 0.56 & 0.85 & 5 \\
\hline 0 and 1 & -0.22 & 0.58 & 0.05 & 0.28 & 0.89 & 30 & 0.34 & 0.64 & 0.05 & 0.69 & 0.55 & 2 \\
\hline 2 & -0.39 & 0.72 & 0.03 & 0.56 & 0.93 & 5 & 0.47 & 0.74 & 0.05 & 0.64 & 0.82 & 5 \\
\hline
\end{tabular}

the staining-only group (1) was very small and similar in lesion distribution to the no staining group $(0)$, these were combined in the analysis of diagnostic parameters (table 4). Correlations and $A_{z}$ values were lower in groups 0 and 1 compared with the entire sample and group 2. Figure 3 shows the corresponding average ROC curves for the whole sample and the staining groups for ECM and CRM.

\section{Discussion}

The histological validation in this study was performed on hemisected teeth. This has been suggested in a study in which the number of occlusal lesions found on hemisected and subsequently serially sectioned teeth was compared [Hintze and Wenzel, 2003]. The dentin caries prevalence in the study was $13 \%$ higher when using serially sectioned teeth ( 7 lesions in 53 teeth), but this was not statistically significant. In our study we chose hemisections, complemented with extra sections when this was deemed necessary for reasons of efficiency. As we made extra sections in case of doubt, we expect that the percentage of lesions missed in our study is lower than in the comparison study. The effect a small number of missed lesions has on diagnostic parameters is not clear, although it may be expected that SE is overestimated and SP is slightly underestimated. Both methods are expected to have been affected in the same way.

The electrical resistances registered in this study appear to be higher than reported before. Although wide variation can be seen in ECM cut-offs reported in the literature - from $\sim 2 \mathrm{M} \Omega$ [Ricketts et al., 1997a] to $7.5 \mathrm{M} \Omega$ [Verdonschot et al., 1993b] - our overall value of 20$25 \mathrm{M} \Omega$ is outside this range. For the CRM there are no literature reports, but our optimal cut-offs lie at higher resistances than the manufacturer suggests, and higher than those of the surface-specific application of ECM, where a surface is dried and conducting medium is applied before taking a single measurement. The unclear optimal cut-offs and the fact that we chose the cut-off with the highest specificity when in doubt, favoring higher resistance values, can partly explain this. It can also be speculated that the embedding of the tooth roots left relatively little surface area for electrical conductance, thus raising resistance values, irrespective of carious involvement. Correlation with carious involvement is not expected to have been compromised.

The average CCCs of the devices indicated only fair reproducibility. Furthermore, the computed limits of agreement derived from the Bland and Altman analysis showed a wide range between measurements. Previous studies on the ECM reported ranges of only $28 \%$ [Ricketts et al., 1997a, b] to 50-55\% [Ekstrand et al., 1997; Pereira et al., 2001] on a 14-unit measurement scale. Our results match the last reports and are considered disappointing. In the repeated measurements it was observed that maximum measurements ( $99 \mathrm{M} \Omega$ ) occurred frequently, and combinations of a measurement somewhere in the measurement range with a maximum measurement were commonplace. The selection of measurement sites as well as the positioning and tilting of the measurement tip could be sources of error. Notably, reaching a stable resistance reading with the ECM was often problematic. This problem has been more extensively described recently [Huysmans et al., in press]. The angular probe tip, combined with narrow fissures and intense drying, may well lead to insufficient contact, and thus maximum resistance readings. In comparison to $\mathrm{ECM}$, the $\mathrm{CRM}$ instrument tended to have a slightly better reproducibility.

The correlation with histological lesion depth revealed nonoptimal values: -0.5 for ECM and 0.6 for CRM. The 
histological extremes - sound dentin and deep dentin lesions - appeared to be essential in establishing a good correlation. For the ECM, the middle categories of histology showed the previously mentioned tendency to yield maximum resistance values (fig. 1a), thus compromising correlation. CRM, like surface-specific ECM, is less prone to such 'sound' extremes (fig. 1b). Still, generally speaking the variation in resistance values within each histological category is large, leading to low correlation.

The $A_{z}$ values registered were below 0.8 for both devices. Previously published $A_{z}$ values for the ECM at the D3-4 threshold were found to be slightly higher [Verdonschot et al., 1993a; Ricketts et al., 1997b; Huysmans, 1998a, b; Ellwood \& Côrtes, 2004]. In general, CRM tended to give slightly better results than the ECM.

In a recent study a newer model of the ECM (ECM IV) has been used with several different techniques, in order to evaluate the impact of the technique on diagnostic performance [Ellwood and Côrtes, 2004]. First of all the basic technique of coaxial drying has been modified to a maximum of $5 \mathrm{~s}$, instead of drying until a stable reading is reached. Also, a similar technique as described here for the CRM was used: drying the occlusal surface for 5$10 \mathrm{~s}$ with air, and subsequently scanning the fissure with the ECM probe. Rank correlation with histological lesion depth for both techniques was low: 0.48 for the $5 \mathrm{~s}$ drying method, and 0.50 for the pre-drying and scanning method. However, $\mathrm{A}_{\mathrm{z}}$ values for dentin caries detection were slightly higher than in our study: 0.86 and 0.84 , respectively. The optimal cut-offs in that study were high, especially for the pre-drying and scanning method. Theoretically, it may be doubted whether resistance measurements higher than about $2 \mathrm{M} \Omega$ are relevant for caries detection, and whether such measured resistances do not reflect a failing probe contact.

Surprisingly, the effect of stained fissures and discolored lesions on diagnostic performance and resistance levels was opposite to that reported by Côrtes et al. [2003]. First of all they reported an increase in the electrical resistance and higher cut-offs needed for stained/discolored lesions, whereas we found lower resistance and cut-offs. It must be stated that the determination of optimal cutoffs was difficult in our study, due to the flat curves. A second observation of Côrtes et al. [2003] was that the correlation with histology was lower for the stained/discolored subgroup than for unstained teeth. Again, we observed the opposite. We attribute this difference to the differences in histological distribution within the subgroups in the two studies. Whereas they found the majority of brown spot lesions in the sound and enamel lesion categories, in our study 42 out of 55 brown discolored lesions were in the dentin lesion categories (table 3). It has already been observed how important the deep dentinal lesion category is for establishing a good correlation of the resistance values with histology. In our sample the lesions in this category were almost all discolored. This should serve to illustrate how dangerous it is to draw conclusions on small and possibly nongeneralizable tooth samples. Whether discoloration of lesions in itself affects electrical caries measurement accuracy is still open to discussion.

It became evident at the study end that the occlusal surfaces had been damaged by the repeated measurements. Both probes, but especially the CRM probe, are sharp and although the manufacturer recommends use without any pressure this is difficult to combine with appropriate electrical contact. Based on these empirical findings, further development of probe configuration may be advisable.

In conclusion, in this in vitro study the CRM showed equal to better diagnostic performance compared with the ECM. Although its diagnostic performance is still not satisfactory, this prototype is a candidate for further development, due to its simplicity and easy use. However, probe tip configuration should be changed in order to limit damage to fragile lesion surfaces. Discolored lesions were shown to result in a different relationship between electrical resistance measurements and histological lesion depth. However, as this effect was opposite to an earlier report, and could be shown to possibly originate in differences in the distribution of histological lesion depth of the samples, the effect of discoloration is still uncertain. 


\section{References}

Axmann D, Gomez G, Groten M: Der Bland und Altman Plot - eine einfache graphische Methode zur Einschätzung der Verlässlichkeit von Messverfahren. Dtsch Zahnärztl Z 2002;57: 613-616.

Bamzahim M, Shi XQ, Angamar-Mansson B: In vitro Comparison of DIAGNOdent und Electronic Caries Monitor for Detection and Quantification of Occlusal Caries. Caries Res 2002; 36:189.

Bland JM, Altman DG: Statistical methods for assessing agreement between two methods of clinical measurement. Lancet 1986;ii:307308.

Côrtes DF, Ellwood RP, Ekstrand KR: An in vivo comparison of a combined FOTI/Visual examination of occlusal caries with other diagnostic methods and the effect of stain on their diagnostic performance. Caries Res 2003;37:816.

Ekstrand KR, Ricketts DNJ, Kidd EAM: Reproducibility and accuracy of three methods for assessment of demineralization depth of the occlusal surface: an in vitro examination. Caries Res 1997;31:224-231.

Ellwood RP, Côrtes DF: In vitro assessment of methods of applying the Electrical Caries Monitor for the detection of occlusal caries. Caries Res 2004;38:45-53.

Gente M: Cariometer CRM 800. Provisional manual and specifications, Marburg, 1999.

Hintze H, Wenzel A: Diagnostic outcome of methods frequently used for caries validation. Caries Res 2003;37:115-124.

Huysmans MCD: Electrical measurements for early caries detection; in Stookey GK (ed): Early Detection of Dental Caries. II. Proc 4th Annu Indiana Conf, Indianapolis, 2000, pp 123143.
Huysmans MCD, Longbottom C, Hintze H, Verdonschot EH: Surface-specific electrical occlusal caries diagnosis: reproducibility, correlation with histological lesion depth, and tooth type dependence. Caries Res 1998a;32:330336.

Huysmans MCD, Longbottom C, Pitts NB: Electrical methods in occlusal caries diagnosis: an in vitro comparison with visual inspection and bite-wing radiography. Caries Res 1998b;32: 324-329.

Huysmans MCD, Kühnisch J, ten Bosch JJ: Reproducibility of electrical caries measurements: a technical problem? Caries Res, in press.

Lin LIK: A concordance correlation coefficient to evaluate reproducibility. Biometrics 1989;45: 255-268.

Lode Diagnostics BV: Operator Manual Electrical Caries Monitor III, Groningen, 1996.

Longbottom C, Huysmans MCD, Pitts NB, Bruce PG: Detection of dental decay and its extent using a.c. impedance spectroscopy. Nat Med 1996;2:235-237.

Lussi A, Firestone A, Schoenberg V, Hotz P, Stich $\mathrm{H}$ : In vivo diagnosis of fissure caries using a new electrical resistance monitor. Caries Res 1995;29:81-87.

Pereira AC, Verdonschot EH, Huysmans MCD: Caries detection methods: can they aid decision making for invasive sealant treatment? Caries Res 2001;35:83-89.

Pine CM, ten Bosch JJ: Dynamics of and diagnostic methods for detecting small carious lesions. Caries Res 1996;30:381-388.

Pitts NB: Clinical diagnosis of dental caries: a European perspective. J Dent Educ 2001;65:972978.

Pitts NB, Stamm JW: Caries research implications of the international collaborative workshop on caries clinical trials. Caries Res 2002;36:208.
Ricketts DNJ, Kidd EAM, Liepins PJ, Wilson RF: Histological validation of electrical resistance measurements in the diagnosis of occlusal caries. Caries Res 1996;30:148-155.

Ricketts DNJ, Kidd EAM, Wilson RF: The effect of airflow on site-specific electrical conductance measurements used in the diagnosis of pit and fissure caries in vitro. Caries Res 1997a;31:111-118.

Ricketts DNJ, Kidd EAM, Wilson RF: The electronic diagnosis of caries in pits and fissures: site-specific stable conductance readings or cumulative resistance readings? Caries Res 1997b;31:119-124.

Ricketts DNJ, Kidd EAM, Wilson RF: Electronic diagnosis of occlusal caries in vitro: adaptation of the technique for epidemiological purposes. Community Dent Oral Epidemiol 1997c;25: 238-241.

Schulte A, Pieper K: Elektrische Widerstandswerte in Fissuren kariesfreier Prämolaren von Erwachsenen und Kindern. Dtsch Zahnärztl Z 1997;52:741-744.

Verdonschot EH, Wenzel A, Bronkhorst EM: Assessment of diagnostic accuracy in caries detection: an analysis of two methods. Community Dent Oral Epidemiol 1993a;21:203-208.

Verdonschot EH, Wenzel A, Truin GJ, König KG: Performance of electrical resistance measurements adjunct to visual inspection in the early diagnosis of occlusal caries. J Dent 1993b;21: 332-327.

Wenzel A, Larsen MJ, Fejerskov Ø: Detection of occlusal caries without cavitation by visual inspection, film radiographs, xeroradiographs, and digitized radiographs. Caries Res 1991;25: 365-371. 